\title{
MONTE CARLO SIMULATIONS OF PERIODIC PULSED REACTOR WITH MOVING GEOMETRY PARTS
}

\author{
Yan Cao and Yousry Gohar \\ Nuclear Engineering Division, Argonne National Laboratory \\ 9700 South Cass Avenue, Argonne, IL 60439 \\ ycao@anl.gov; gohar@anl.gov
}

\begin{abstract}
In a periodic pulsed reactor, the reactor state varies periodically from slightly subcritical to slightly prompt supercritical for producing periodic power pulses. Such periodic state change is accomplished by a periodic movement of specific reactor parts, such as control rods or reflector sections. The analysis of such reactor is difficult to perform with the current reactor physics computer programs. Based on past experience, the utilization of the point kinetics approximations gives considerable errors in predicting the magnitude and the shape of the power pulse if the reactor has significantly different neutron life times in different zones. To accurately simulate the dynamics of this type of reactor, a Monte Carlo procedure using the transfer function TRCL/TR of the MCNP/MCNPX computer programs is utilized to model the movable reactor parts. In this paper, two algorithms simulating the geometry part movements during a neutron history tracking have been developed. Several test cases have been developed to evaluate these procedures. The numerical test cases have shown that the developed algorithms can be utilized to simulate the reactor dynamics with movable geometry parts.
\end{abstract}

Key Words: periodic pulsed reactor, Monte Carlo method, movable reactor geometry parts

\section{INTRODUCTION}

In the $50 \mathrm{~s}$ to $70 \mathrm{~s}$ of last century, several nuclear pulse reactors were built worldwide for studying reactor physics, nuclear material performance, etc. [1]. These reactors are designed to operate with short power pulses. Based on the used procedure for generating the power pulses, there are three types of nuclear pulse reactors: Fast Burst Reactors (FBR), Periodic Pulsed Reactor (PPR), and Booster or the so called source driven system.

The power pulses in a FBR usually are initiated by the rapid insertion of excess reactivity for transitioning the reactor to a super prompt critical state then the negative temperature feedback quenches the excess reactivity. The US Lady Godiva and later the TRIGA are examples of such burst reactors, which only produce a single power pulse each time. For the PPR, the power pulses are mainly formed due to the modulation of the reactivity with a specific periodicity. The reactor is modulated externally and its state varies from subcritical to prompt supercritical to produce a power pulse then back to subcritical for removing the excess reactivity. The Russian IBR-2 reactor [2] is an example of a periodic pulsed reactor for producing multiple power pulses. It has been successfully operated for many decades. For boosters, the acceleratordriven subcritical system is one of the examples. The reactor stays at a subcritical state all the time while the power pulses are generated from the neutron multiplications of external neutrons. Usually, the external neutron source has D-D or D-T neutrons from Deuteron accelerator with Deuteron or Tritium target, respectively; or spallation neutrons from Proton accelerator with a target using high atomic number material. 
This paper examines a PPR, which has movable geometry parts for generating neutron pulses. During the operation, the movable geometry parts move in and out of the reactor core changing the reactor state from a slightly prompt supercritical to a slightly subcritical. The dynamics of this type of reactors is difficult to simulate with the current reactor physics computer programs. According to past experiences, the point kinetics approximations can only provide approximate results when the PPR core is tight and "homogeneous" in terms of the neutron lifetimes. For multi-zones reactor with different neutron mean lifetimes for each zone, i.e., a reflector with light materials in a fast reactor, the point kinetics approximations give considerable errors in predicting the power and the pulse shapes. Using a more complicated multi-point and multi-energy group kinetics model, the agreement between the numerical simulations and the experiments are slightly improved [1].

To simulate accurately the dynamics of this type of reactors, Monte Carlo simulation models of the different reactor zones are considered. In this paper, a numerical procedure which utilizes the Monte Carlo method to simulate the dynamics of a PPR reactor is presented. In particular, a procedure of simulating movable geometry parts using the transfer functions of the MCNP/MCNPX computer program is discussed [3]. Several test cases are setup to verify the extended capabilities of the Monte Carlo methods using MCNP/MCNPX.

\section{MONTE CARLO MODELING OF A REACTOR WITH MOVING GEOMETRY PARTS}

\subsection{Modeling of the Geometry Part Movement}

Monte Carlo method is very powerful for simulating the complex geometry of a nuclear reactor. For example, MCNP/MCNPX uses first- and second-degree surfaces or fourth-degree elliptical tori to define a three-dimensional geometrical cell. The cell can be further defined by the intersections, unions of cells or complements of the regions [3]. MCNP/MCNPX has a lot of features such as cell/surface transfer, lattice, or universe to help model different geometries. PPR cores have irregular geometries, and their geometries can be described using the current capabilities of MCNP/MCNPX. However, the MCNP/MCNPX fixes the geometry description and material properties before starting any tracking process. The surface coefficient arrays are pre-calculated and stored for the particle tracking process.

To model the movable geometry parts along the particle tracking, first, the movement of the geometry parts needs to be addressed and should be defined in the numerical simulation. In other words, at any time, the positions of the movable geometry parts can be determined. Therefore, the positions of the geometry parts can be calculated based on the particle history time (t). An extra subroutine is developed to calculate these instant positions of the moving parts at time $t$ assuming the parts are moving with constant velocity.

In this subroutine, the surface/cell transfer function (TRCL card) in MCNP/MCNPX computer program is utilized to move the geometry parts to the right position at any given time. A time-dependent transfer function is defined to describe the trajectory of the movable geometry parts. The calculated time dependent transfer coefficients are supplied to the MCNP/MCNPX code to update the surface coefficient arrays. Since the particle history time is a variable, to be consistent, all the transfer coefficients are always calculated with respect to the initial position before moving the geometry parts. The flow chart in Figure 1 illustrates the implementation procedure. As shown in the picture, the procedure left all the cells without movable geometry 
parts unperturbed. The additional computation of this procedure is small relative to the overall computational time.

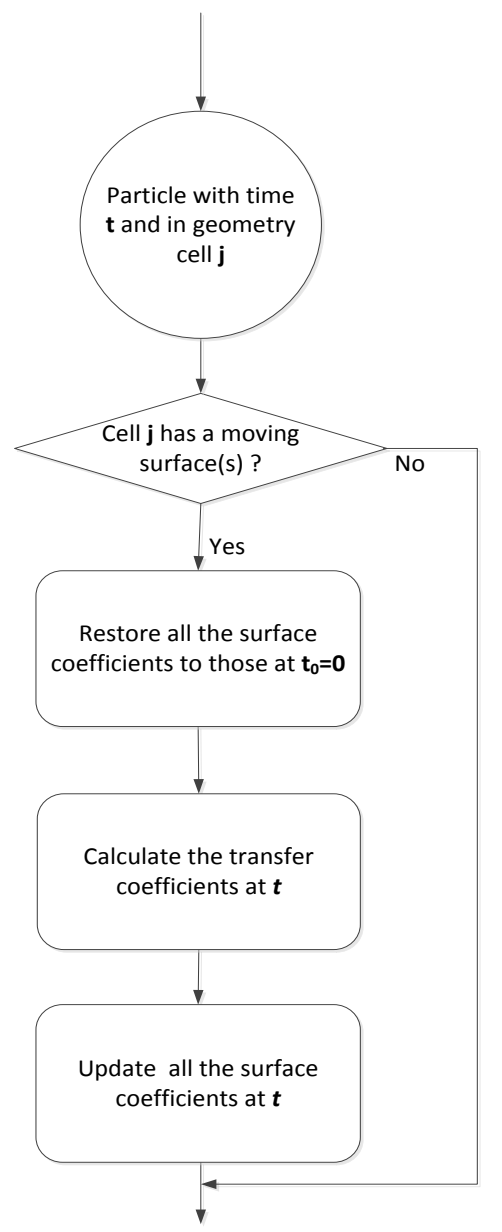

Figure 1. Flow chart for modeling the movable geometry parts.

\subsection{Monte Carlo History Tracking with Moving Geometry Parts}

In the MCNP/MCNPX random walk, the track length of the particle moving from one position to another is the minimum of several calculated distances in the particle direction: to the next collision site, to the nearest cell boundary, to the nearest DXTRAN sphere, to time cutoff, and to the weight window cell boundary. If the selected tracking distance is to a DXTRAN sphere, to a time cutoff, or to a weight window cell boundary in the particle direction, the particles will be terminated or a Russian roulette survival game is played. The particle undergoes a collision if the selected tracking distance is to the next collision site. If the selected tracking distance is to surface crossing, the particle is moved to cross that surface and it is prepared to enter the next adjacent cell. In all the cases, the particle's new parameters are updated at the end of the history track. The Monte Carlo simulation calculates the requested tallies, and is ready for another tracking step from the new location [3].

To address the movement of the geometry parts during the history tracking, some extra steps are used. Namely, during the time period that the particle transports from the current position to the next position, the movable geometry parts have changed their positions slightly. To take 
account of these movements in the random walk, the first simple way is to assume that the movements of the geometry parts are very small and can be neglected because the moving surfaces speeds are negligible relative to the particle speed. Therefore, the movable geometry parts only change the positions before each particle event and remain at the same positions until the end of this event. Figure 3 (a) and (b) illustrates the differences of the modified particle history track using this simple method. The surface coefficients corresponding to the movable geometry parts are updated at the beginning of each tracking step. Due to the shift of the cell/surface before starting the new track step, it is possible that some of the particles might be positioned in a wrong cell. As shown in Figure 3 (b), extra steps are also used to place the particle into the right cell before the next tracking step.

The simple method assumes the geometry part moving speed is much smaller than the speed of the tracked particle so that its movement can be treated as adiabatic steps. When the parts move with a speed comparable to the particle speed, the simple method can lead to noticeable errors. An improved and more accurate approach is to move the geometry parts during the history tracking step. In this method, the distance to the nearest surface boundary is calculated using the relative speed between the particle and the moving geometry parts. Theoretically, for simple cases, the relative speed can be calculated if the surface movement is pre-determined. However, due to the random directions of each history track and for some complex reactor geometry, it is difficult to find simple analytical solutions which can quickly calculate the relative speed. In this paper, an approximate approach which only requires two additional steps is adopted. Figure 3 (c) illustrates the modified history flow of this method.

In the improved method, similar to the simple method, the position of the movable geometry parts is first updated at the beginning of the history tracking step with particle history time $t_{1}$ and particle velocity $V_{n}$. A minimum distance $d$ is obtained by tracing the particles to the next surface, to the collision site, etc. as previously discussed. In addition, for each surface $i$ defining the current geometrical cell, the distance $d_{1, i}$ of the particle to the $i$ surface along the particle moving direction is recorded. For the time interval that the particle takes to travel the minimum distance $d$, the movable geometry parts are moved to a new location. This new location can be calculated using the time $t_{2}=t_{1}+d / V_{n}$ and the surface coefficient arrays are updated accordingly. Then the distance $d_{2, i}$ to the updated surface position is calculated. With these two distances, the speed of each moving surface along the particle history track can be estimated assuming that the surface only moves in one direction during this short time interval $d / V_{n}$ :

$$
V_{s, i}=\frac{d_{2, i}-d_{1, i}}{d} V_{n}
$$

As a result, the distance of the particle to each surface can be calculated more accurately as $d_{3, i}$ using the calculated surface speed $V_{s, i}$ :

$$
d_{3, i}=\frac{V_{n}}{V_{n}-V_{s, i}} d_{1, i}
$$




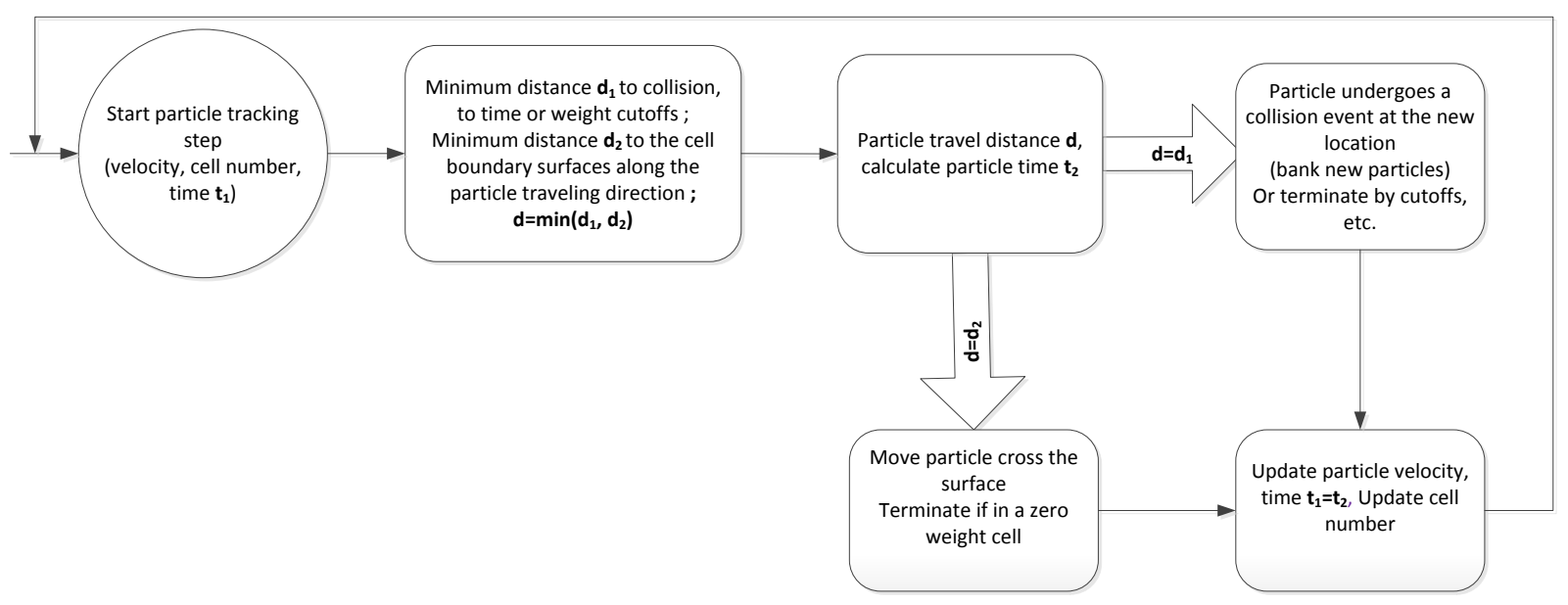

(a)

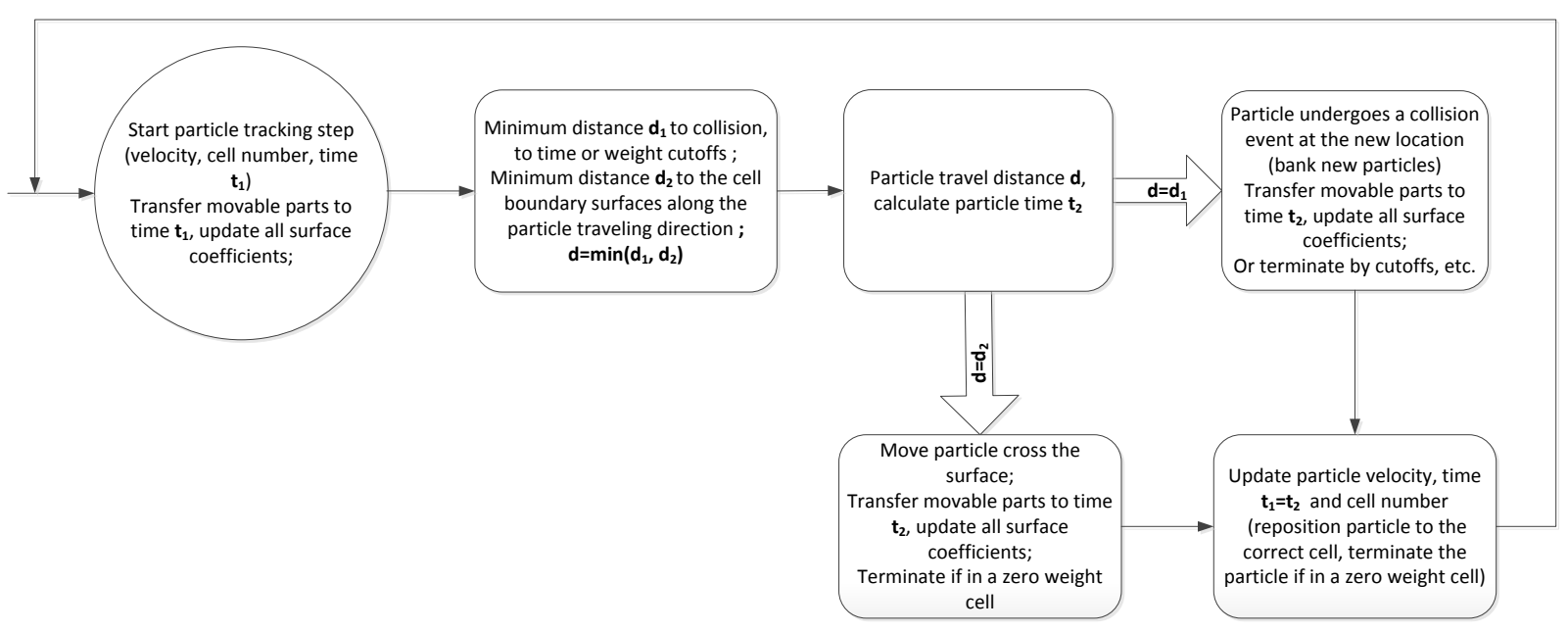

(b)

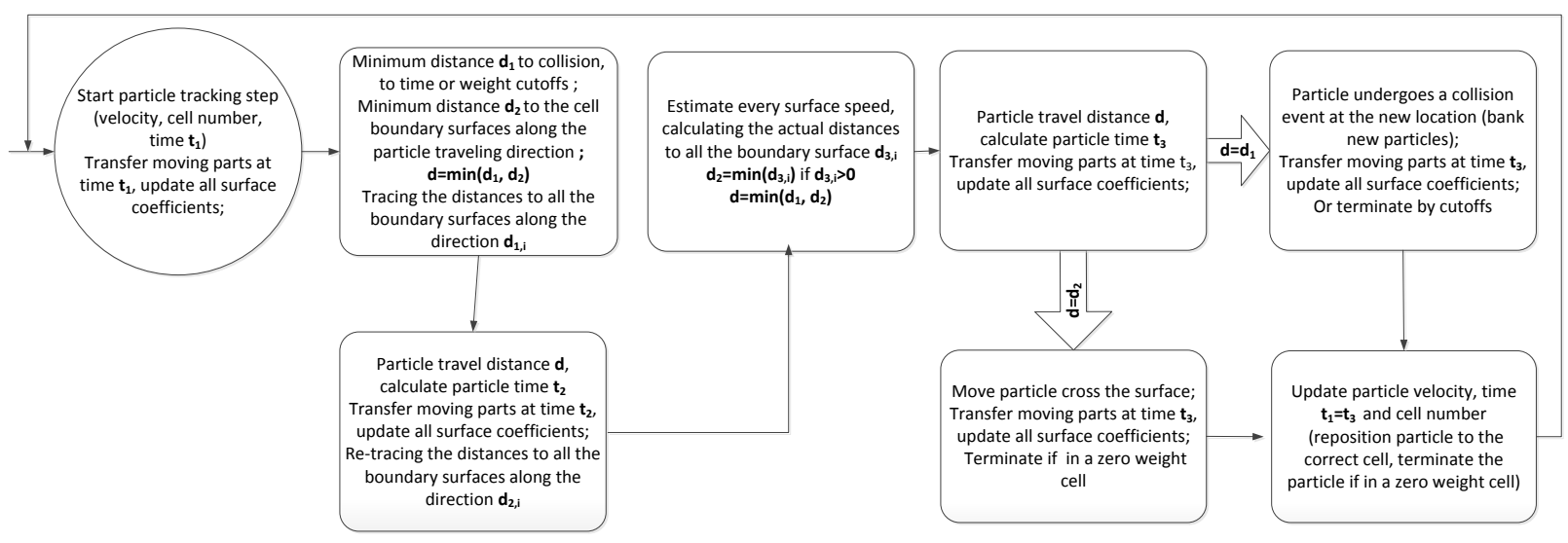

(c)

Figure 3. Particle history tracking flow chart in MCNP/MCNPX code (a) original (b) simple method for moving geometry part, and (c) improved method of moving geometry part. 
Then, the actual distance to surface crossing can be obtained as the minimum distance of all the $d_{3, i}$. It is compared with all the other distances previously sampled, such as the distance to next collision site, to time cutoff and etc. The rest of the history tracking is similar to the simple method as shown in Figure 3(c). Overall, the improved method requires two extra steps for tracing the particle in zones containing or next to the moving geometry parts. It takes into account the movement of the surfaces within the history tracking step. Test cases with simple geometries and movable parts are setup to compare both methods.

\section{TEST CASES}

\subsection{Null Transient Case}

The first test case is a null transient, which simulates a steady state case. A simple geometry consists of a fuel box on top of a reflector box is used. The fuel box is a $60 \mathrm{~cm}$ cube which has $\mathrm{UO}_{2}$ and aluminum alloy mixture. The reflector is a $220 \mathrm{~cm} \times 90 \mathrm{~cm} \times 30 \mathrm{~cm}$ graphite box positioned under the fuel box as shown in Fig. 4. The graphite box is modeled with two boxes, one inside the other. The inner box dimensions are $70 \mathrm{~cm} \times 70 \mathrm{~cm} \times 30 \mathrm{~cm}$. It moves inside the outer box with both boxes containing the same graphite material. The center of the inner box moves back and forth in the $\mathrm{x}$ direction from $-70 \mathrm{~cm}$ to $+70 \mathrm{~cm}$. The full cycle time starting the initial conditions shown in Fig. 4 is $40 \mathrm{~ms}$. The outer box zone is the complimentary zone of the inner box. Its geometry also changes accordingly as the inner box moves. A point neutron source is positioned in the fuel zone at $0.0,0.0,5.0 \mathrm{~cm}$, which is $5 \mathrm{~cm}$ above the reflector box and inside the fuel box. The source strength is always uniformly sampled over the interested time period.

The average neutron flux inside the fuel box is tallied by three different Monte Carlo simulations. The first simulation is performed using the original MCNP/MCNPX computer program. The reflector zone is modeled as a single zone and fixed under the fuel box. This calculation is a steady state calculation since the reflector is not moving. The second simulation uses the Monte Carlo code with the simple method implemented. The reflector is separated into the inner and outer zone, with inner zone moved back and forth inside the outer zone. The third simulation uses the Monte Carlo code with the improved tracking method for the reflector movement. In this simulation, the inner zone is also moved back and forth inside the outer zone. The latter two simulations are referred as "dynamic calculations" due to the artificial movement of inner graphite zone inside the outer graphite box.

Figure 5 shows the neutron fluxes obtained from the three simulations within the first period. The time period is divided into 400 time bins. During the first few time bins, the neutron populations quickly grow in the fuel box for all the cases, since all neutrons are assumed to be born after the source is initiated at $t=0$ in this model. The neutron fluxes reach a steady state value quickly for three simulations. Clearly, the tallied neutron fluxes from both dynamic calculations match the fluxes from the steady state calculations very well. Figure 6 plots the relative errors of the dynamic neutron fluxes to the steady state neutron fluxes at each time bin. It shows that both dynamic neutron fluxes agree with the steady state fluxes within the statistic error of $0.2 \%$, and no biases are observed in the numerical simulations for both dynamic neutron fluxes.

Monte Carlo simulations with inner zone moving at a shorter period of $400 \mu$ s are also performed to compare the results from both methods. Figure 7 shows the calculated relative errors of the dynamic neutron fluxes to the steady state neutron fluxes. No significant 
differences from Figure 6 are observed in Figure 7 as the reflector speed increases. Overall, these numerical test cases verify that in this null transient case, both the simple method and the improved method are accurate in tallying the neutron fluxes.

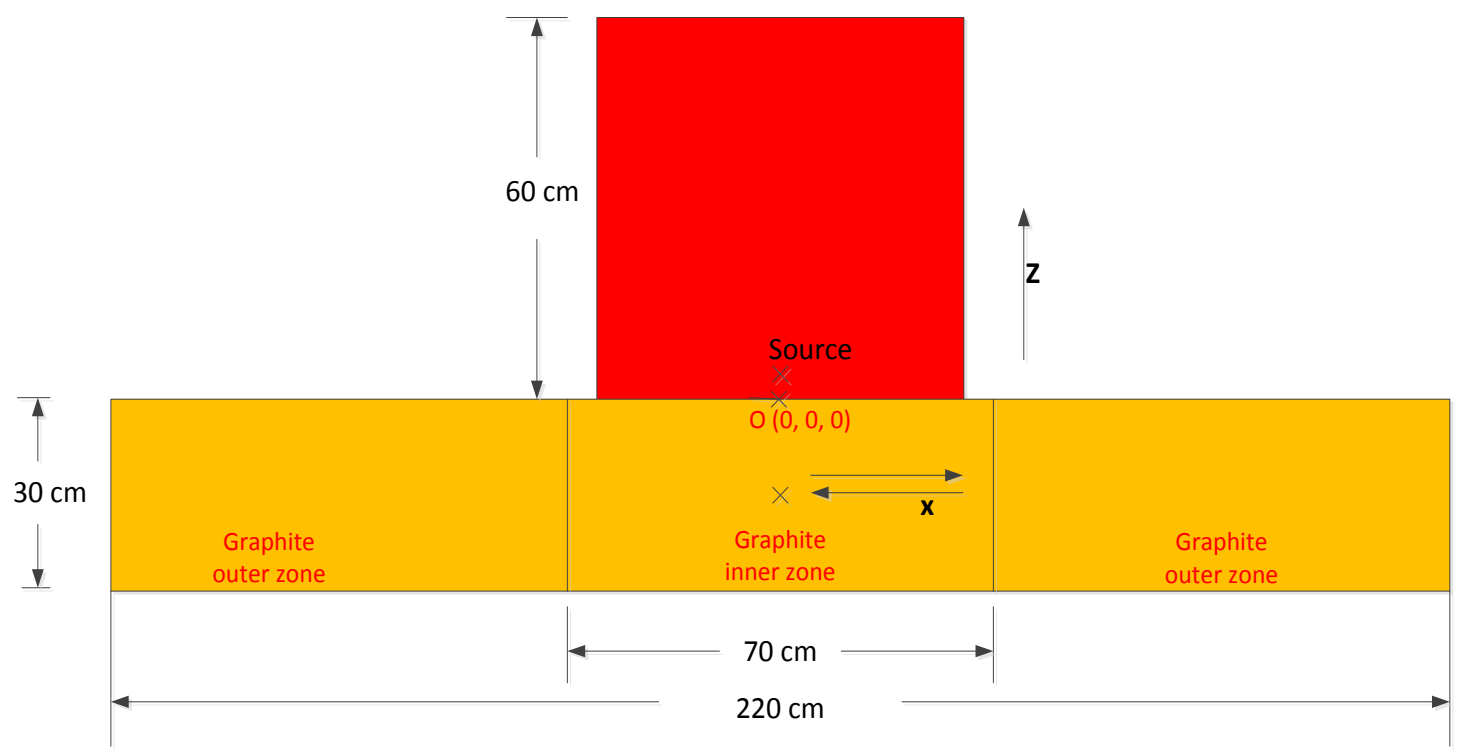

Figure 4. Geometrical model of the null transient test case.

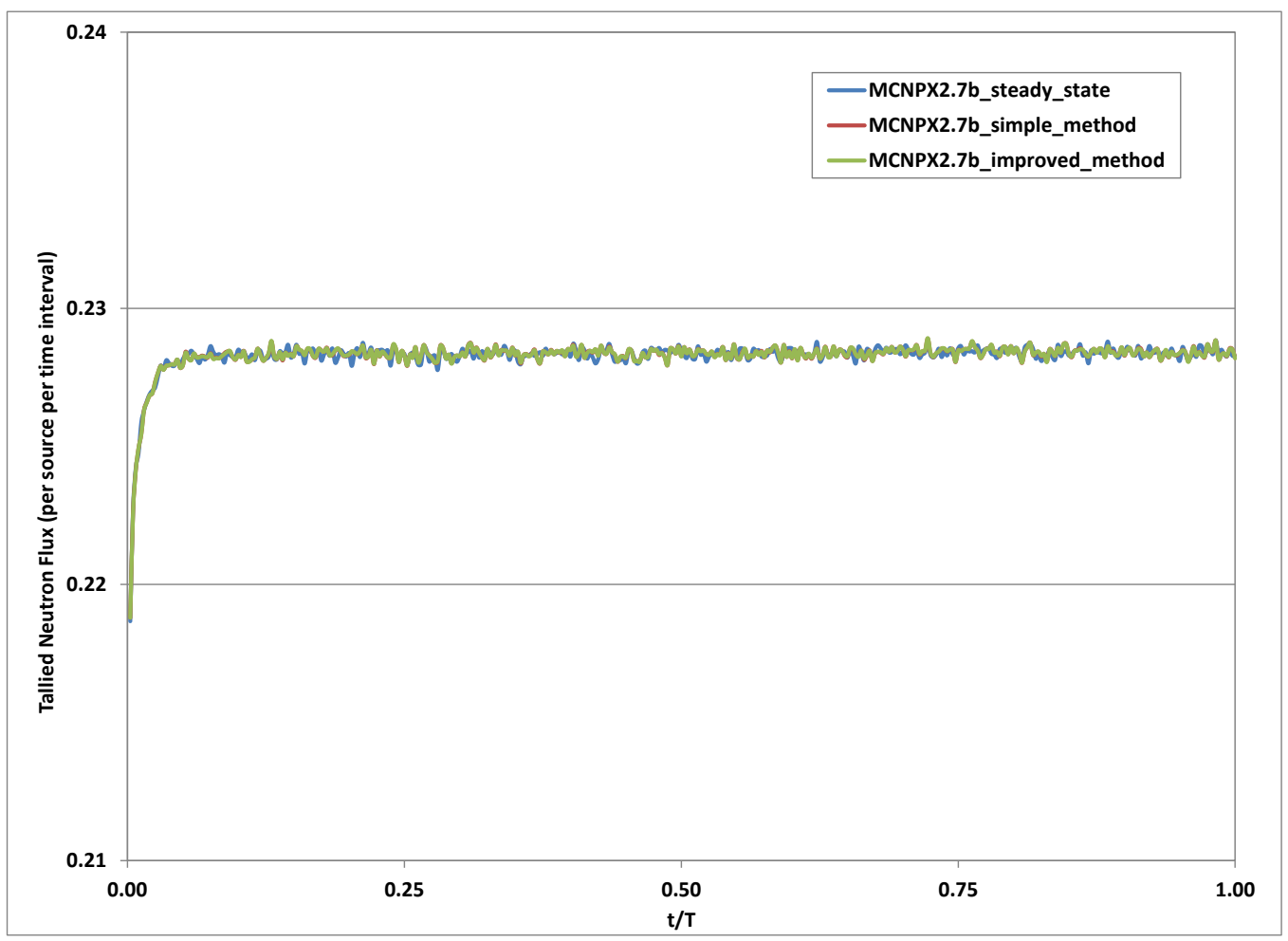

Figure 5. Comparison of the neutron fluxes tallied in the fuel box from the three the Monte Carlo simulations for the null transient case with the reflector inner zone moving period of $40 \mathrm{~ms}$. 


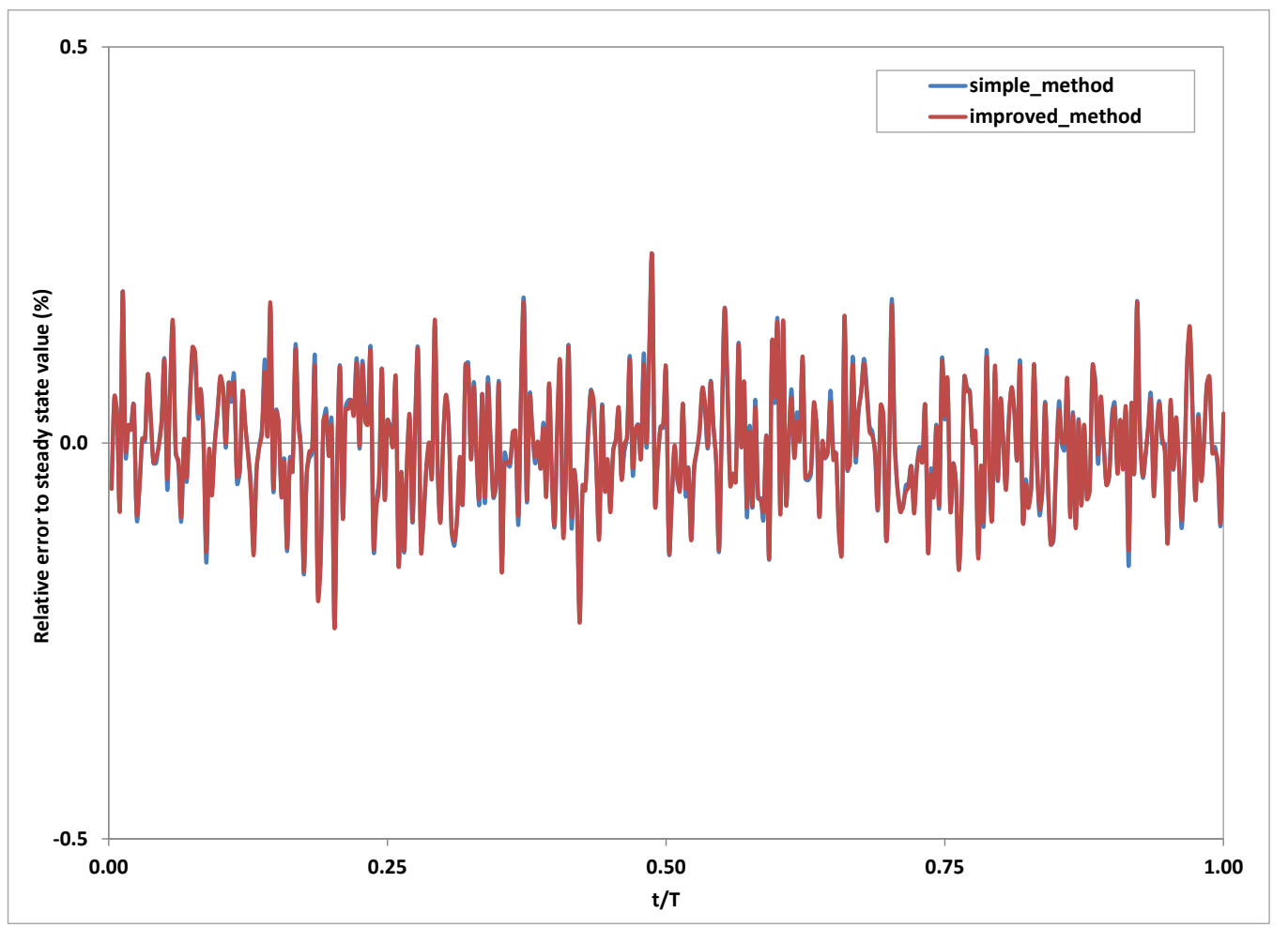

Figure 6. Relative errors of the neutron fluxes calculated using the simple and improved Monte Carlo methods for the null transient case with the reflector inner zone moving period of $\mathbf{4 0 ~} \mathrm{ms}$.

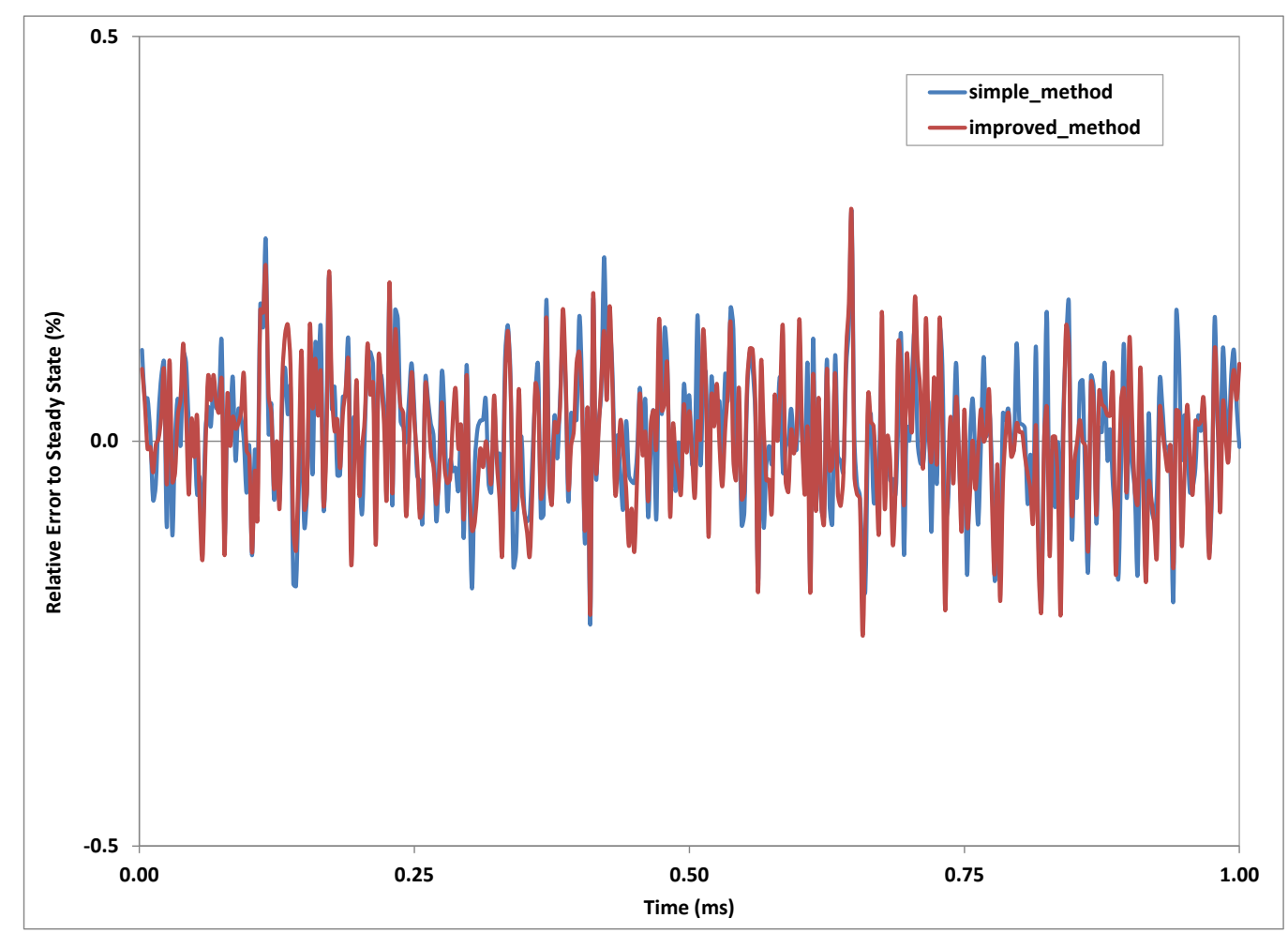

Figure 7. Relative errors of the neutron fluxes calculated using the simple and improved Monte Carlo methods for the null transient case with the reflector inner zone moving period of $400 \mu \mathrm{s}$. 


\subsection{Subcritical Case}

The geometry of the second test case is similar to the null transient case, except the graphite material of the outer zone is replaced with air. Thus, the graphite reflector moves back and forth in the air. Its center moves from the origin at $\mathrm{x}=0 \mathrm{~cm}$ to the far right position $\mathrm{x}=140 \mathrm{~cm}$, and turns back to the far left position $\mathrm{x}=-140 \mathrm{~cm}$, and then returns back to the center position at the end of the period. During this time period, the reflector moves with the same speed in the both directions of the $\mathrm{x}$ axis. The effective multiplication factor $\left(k_{e f f}\right)$ varies from [0.135 0.186] while the graphite reflector changes its position. Figure 8 plots the calculated $k_{\text {eff }}$ and its mean generation time as a function of the movable reflector center position using the MCNP6 simulations [4].

Similarly to the null transient case, the dynamic neutron fluxes in the fuel box are tallied using the Monte Carlo codes with the simple method and the improved method discussed above. The simulations were performed for the first 5 time periods. Each time period is divided into 800 time bins. For the purpose of this analysis, only the prompt neutrons are considered in all the Monte Carlo simulations for this test case. The external source is at the same position as defined in the previous null transient case. It is sampled uniformly during the 5 time periods.

Different from the previous null transient case, there is no steady state solution of the neutron fluxes inside the fuel box due to the real movement of the reflector. Instead, a series of static Monte Carlo fixed source calculations are performed to obtain the static neutron fluxes in the fuel box with the reflector centered at different positions between $\mathrm{x}=-140 \mathrm{~cm}$ to $\mathrm{x}=140 \mathrm{~cm}$. The static neutron fluxes are calculated with the reflector shifted in equal steps of $5 \mathrm{~cm}$ each. Figure 9 plots the static neutron fluxes using the reflector center position converted to the time inside each period. The dynamic neutron fluxes calculated using the Monte Carlo code with the improved method for the first period with different time periods, $20 \mu \mathrm{s}, 2 \mathrm{~ms}, 20 \mathrm{~ms}, 200 \mathrm{~ms}$, and $800 \mathrm{~ms}$. The results shows that when the time period is large, the dynamic neutron fluxes obtained with the improved method agree with the static calculations very well at all these static points. For small time period, the dynamic neutron fluxes start to deviate from the static points.

Figure 10 shows the neutron fluxes obtained from the Monte Carlo dynamic calculations for the first 5 time periods. After the second time period, the time dependent neutron flux reaches a repetitive state. The peak value of the neutron flux decreases significantly for all the cases with the time period less than about $2 \mathrm{~ms}$. For cases with time period less than $2 \mathrm{~ms}$, the reflector moving speed is larger than $2.8 \mathrm{~km} / \mathrm{s}$. In this case, the reflector moves so fast that there is not enough time for prompt neutrons to get reflected inside the fuel box.

The dynamic neutron fluxes obtained with the simple method are compared with the dynamic neutron fluxes obtained with the improved method in Figure 11. Figure 12 shows the same comparison but extends the results to the first five periods. When the time period is large, both dynamic neutron fluxes agree with each other at each time bin. However, when the time period is small, the dynamic neutron fluxes obtained with the simple method gradually deviate from the fluxes obtained with the improved method. Particularly, for all the cases with the time period $\leq 2 \mathrm{~ms}$, the fluxes obtained with the simple method seems larger than those obtained with the improved method in the first quarter of the period. The differences are reduced in the second quarter. The differences are enlarged again in the third quarter and are reduced in the fourth quarter. When the reflector moves extremely fast, i.e., $\mathrm{T}$ is $\sim 0.2 \mu$ s, the fluxes obtained using the simple method are consistently larger than the fluxes obtained using the improved method for 
this test case.

The differences can be explained by examining the assumption used in the simple method that the moving geometry part moves only at the beginning of each history track. With this assumption, for this test case, when the reflector moves away from the fuel box center, the simple method keeps the reflector at a position close to the fuel box with a large $\mathrm{k}_{\text {eff }}$ for longer time than the improved method. Vice versa, when the reflector moves toward the fuel box, the simple method keeps the reflector at a position far from the fuel box with a small $\mathrm{k}_{\text {eff }}$ for longer time. When the reflector period is $\sim 0.2 \mu \mathrm{s}$, the reflector velocity is $\sim 28000 \mathrm{~km} / \mathrm{s}$, which is comparable to the velocity of $20 \%-30 \%$ of the neutron populations in the movable reflector. Thus, the assumption used in the simple method starts to introduce significant errors in some of the history tracks.

\section{CONCLUSIONS}

In this paper, two methods are developed to simulate the dynamics of a nuclear reactor with movable geometry parts. In particular, a procedure is developed to model the movement of the geometry parts using the original transfer function TRCL/TR of the MCNP/MCNPX computer program. Two methods considering the surface movement during the particle tracking process are implemented in the MCNP/MCNPX computer program. A simple method moves only the movable geometry parts at the beginning of each history track event and an improved method estimates the moving surface speed along the particle direction.

Two test cases have been setup using both methods. The first case has a null transient. In this case, the dynamic neutron fluxes calculated using both the simple method and the improved method agree with the steady state neutron fluxes which are calculated by the original MCNP/MCNPX computer program. It shows that both the simple method and improved method are accurate in this null transient case.

The second test case has an actual transient model with the reflector assumed to be moved back and forth under a nuclear fuel box. The dynamic neutron fluxes are calculated with reflector moving at different speeds. A set of static neutron flux points distributed over the time period is obtained using the original Monte Carlo computer program. It was found that the dynamic neutron fluxes calculated by both the simple and the improved method asymptotically approach the static neutron flux points when the reflector velocity is much slower than the neutron speed. The results from both methods match the static results when the reflector moves very slowly.

The second test case also shows that the dynamic neutron fluxes obtained using both methods agree with each other when the reflector moves slowly. However, the differences between these two dynamic neutron fluxes grow when the reflector moves fast. For the particular test case, the neutron fluxes obtained by the simple method are constantly different with those obtained by the improved method if the reflector moves with a speed which is comparable to the neutron speed. The differences are mainly due to the gradually increased errors caused by the assumption used in the simple method. The reflector movement during the history track is not negligible when the reflector moving speed is comparable to the neutron velocity. 


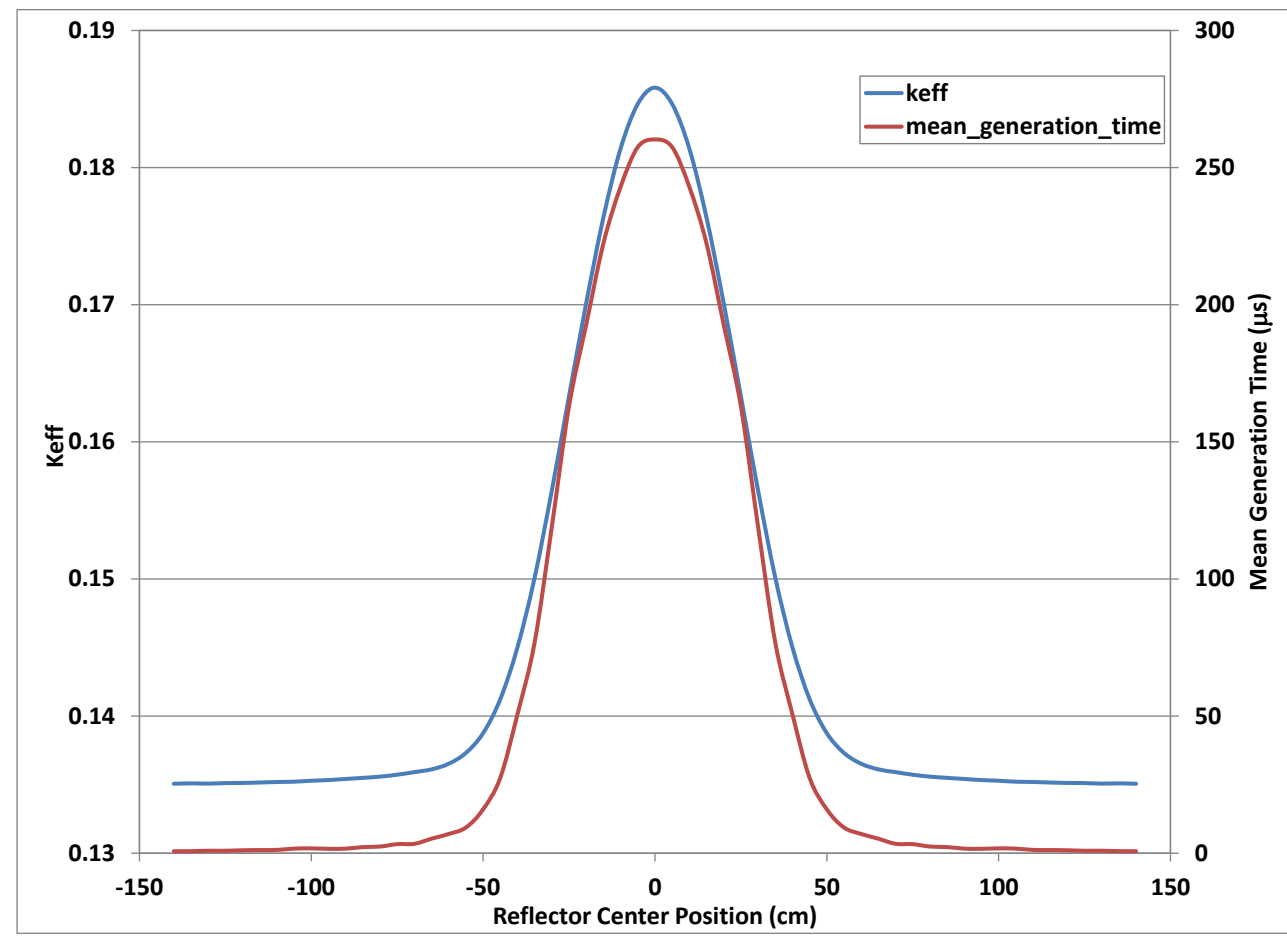

Figure 8. $k_{\text {eff }}$ and mean generation time of the subcritical test case with reflectors fixed at different positions using MCNP6.

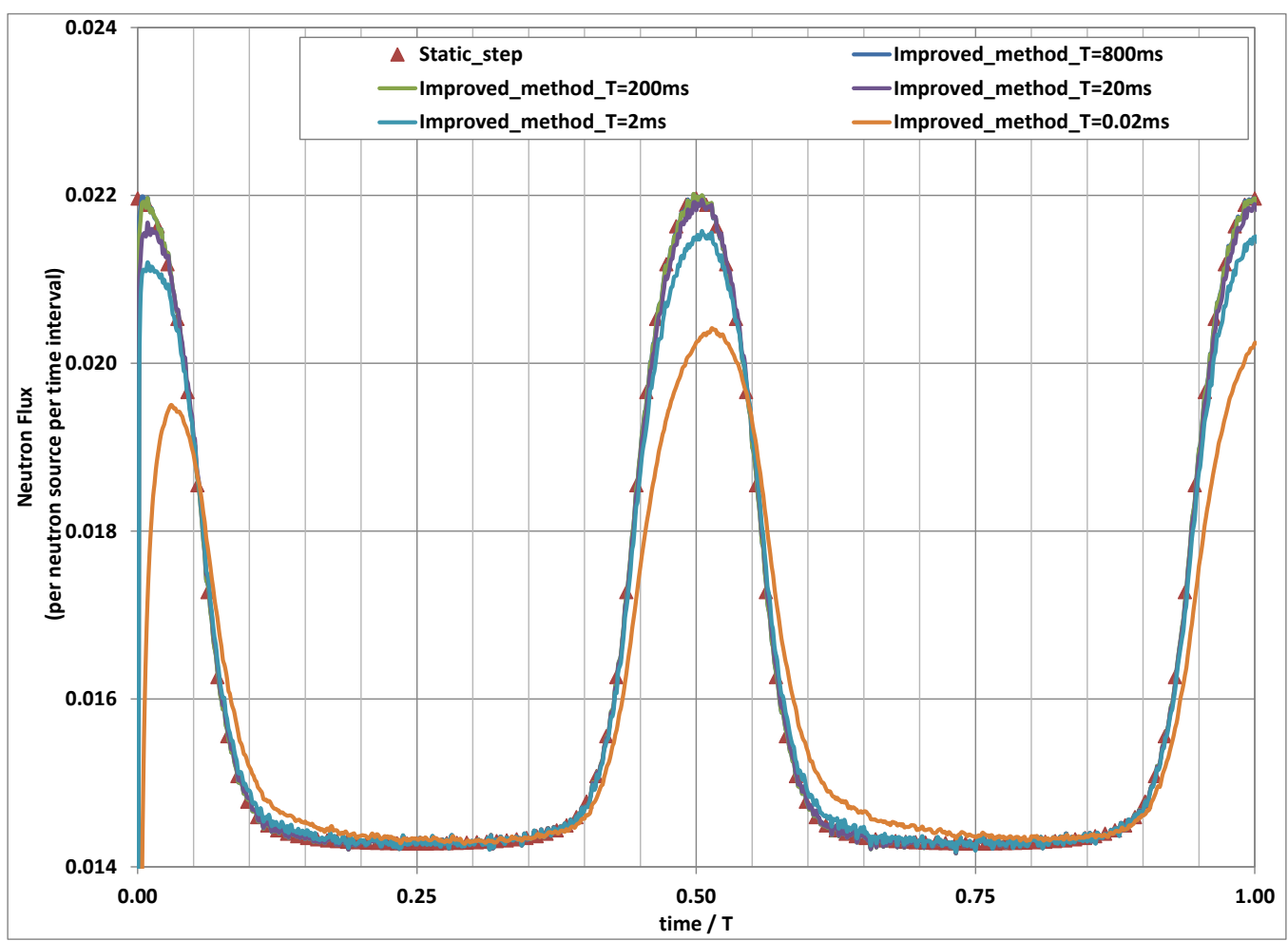

Figure 9. Comparison between the static neutron fluxes and the dynamic neutron fluxes tallied in the fuel box using the improved method with different time periods for the subcritical case. 


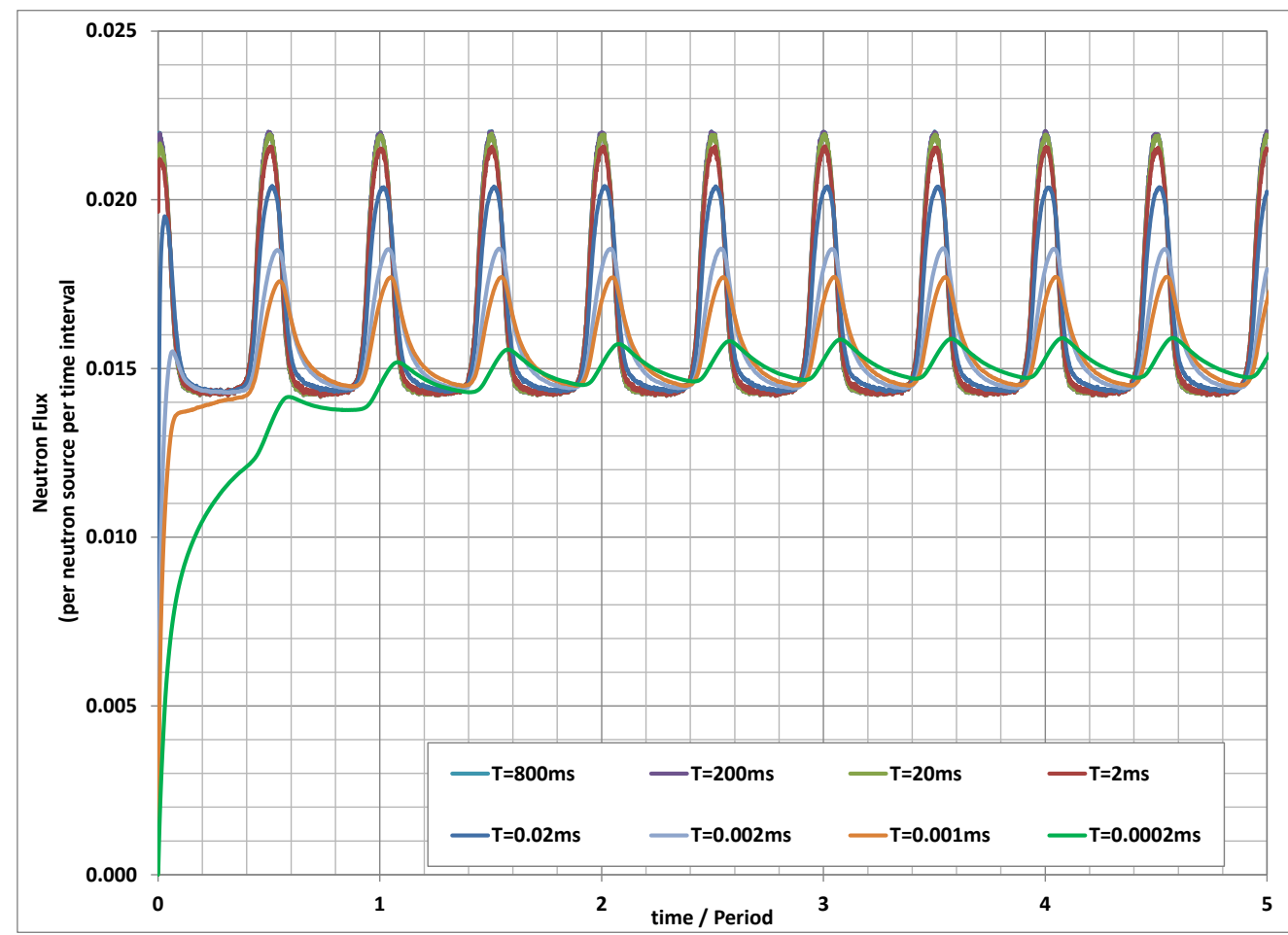

Figure 10. The calculated dynamic neutron fluxes tallied in the fuel box using the improved method for the subcritical case in the first 5 periods with the reflector time period varying from $0.2 \mu$ s to $800 \mathrm{~ms}$.

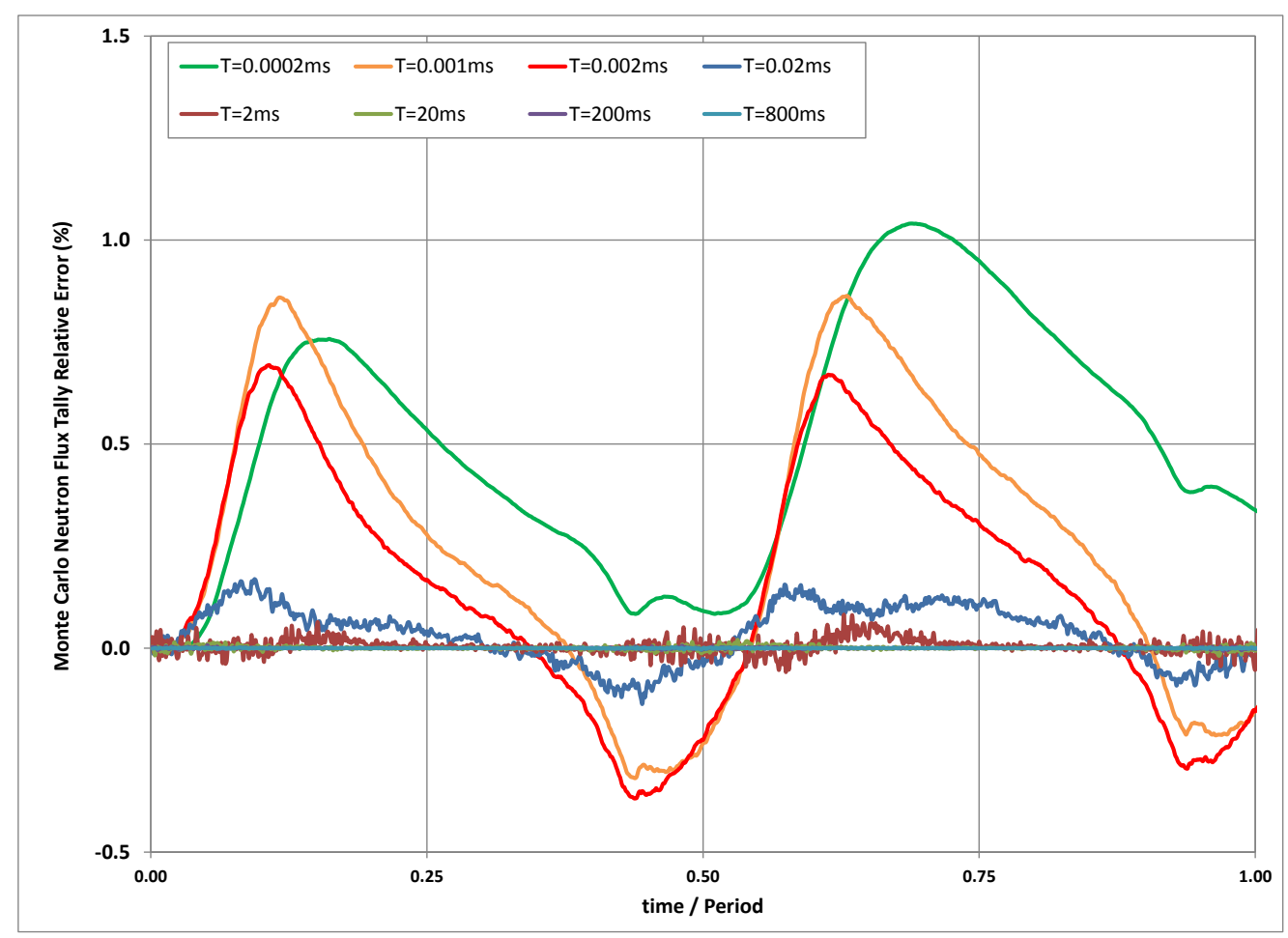

Figure 11. Neutron flux errors of the simple method relative to the improved method for the subcritical case in the first period with different time periods. 


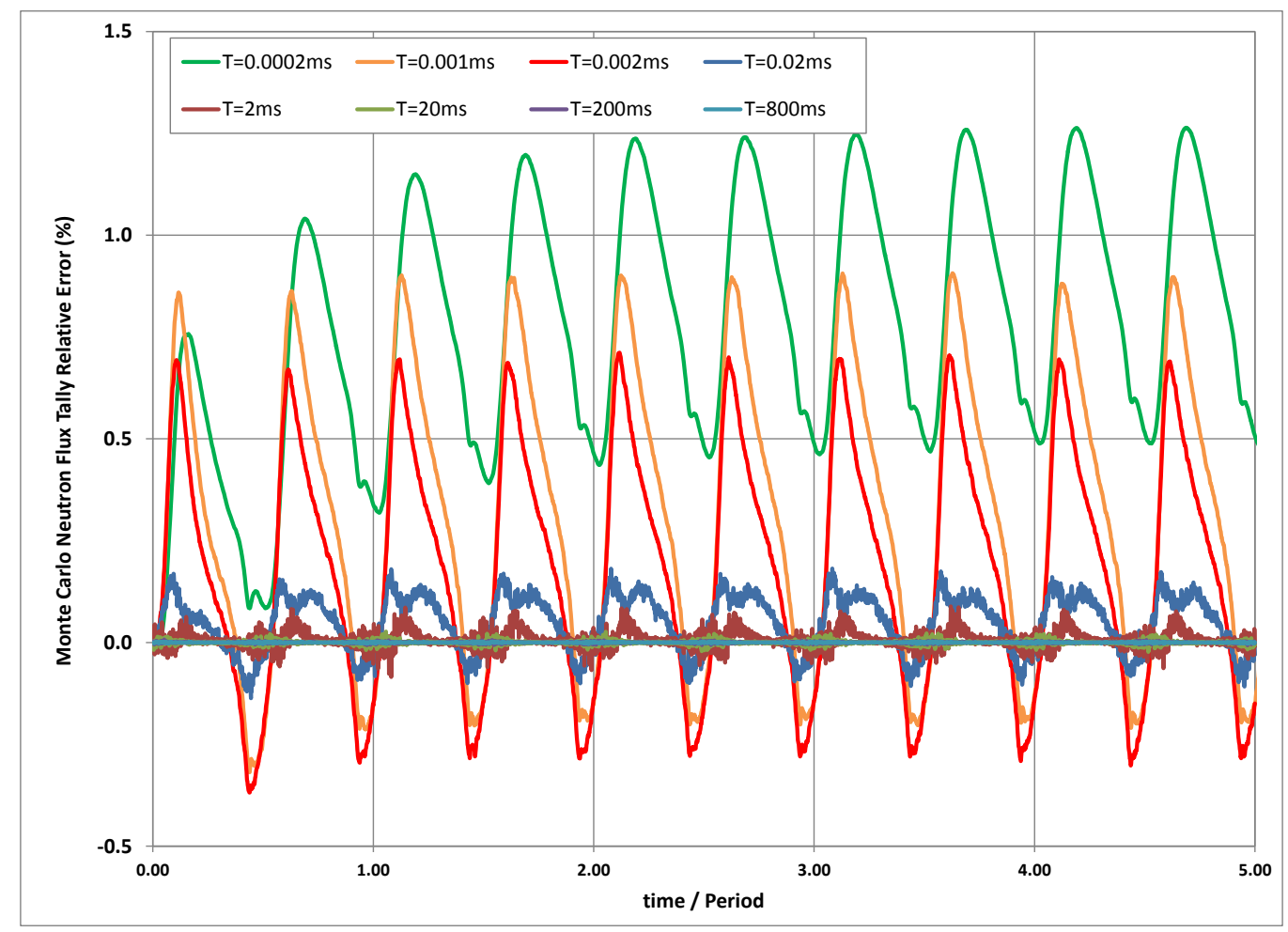

Figure 12. Neutron flux error of the simple method relative to the improved method for the subcritical case for the first five period with different time periods.

\section{ACKNOWLEDGMENTS}

This work has been supported by the Office of Global Nuclear Material Threat Reduction U.S. Department of Energy Under Contract DE-AC02-06CH11357.

\section{REFERENCES}

1. E. P. Shabalin, Fast Pulsed and Burst Reactors, Translated from the Russian: Impulsnye reaktory na bystrykh neitronakh. New York: Pergamon Press (1979).

2. Anatoli Diakov, "On Conversion of Research Reactors in Russia," Center for Arms Control, Energy \& Environmental Studies (2013).

3. D. B. Pelowitz, "MCNPX ${ }^{\mathrm{TM}}$ user's manual," LA-CP-07-1473 (2008).

4. D. B. Pelowitz, "MCNP6 ${ }^{\mathrm{TM}}$ user's manual," LA-CP-11-01708 (2011). 